Short Communication

\title{
Reconstruction of Aberrant Left Hepatic Artery in Orthotopic Liver Transplantation - Single Center Experience
}

Plamen Mihaylov *, Burcin Ekser, Daiki Soma, Andrew Lutz, Jonathan Fridell, Chandrashekhar A. Kubal

Indiana University School of Medicine, Dept. of Surgery, Transplant Division; E-Mails: pmihaylo@iupui.edu; bekser@iupui.edu; daiksoma@iu.edu; ifridell@iupui.edu; alutz@indiana.edu; kubal@iupui.edu

* Correspondence: Plamen Mihaylov; E-Mail: pmihaylo@iupui.edu

Academic Editor: Michel Mourad

Special Issue: Abdominal Organ Transplantation

OBM Transplantation

2021, volume 5 , issue 1

doi:10.21926/obm.transplant.2101138
Received: January 04, 2021

Accepted: March 08, 2021

Published: March 11, 2021

\begin{abstract}
One of the most feared complications in liver transplantation is hepatic arterial thrombosis (HAT). The incidence of HAT in liver transplantation varies from $1.2 \%$ and $8 \%$. One of the risk factors for this complication is anatomical complexity of hepatic arterial system. The focus of this short communication is to show our approach in dealing with aberrant left hepatic artery in settings of liver transplantation. This is a single center experience. Between January 2016 and June 2019, we procured and transplanted 357 adult liver allografts. Of these, there were 34 (9.5\%) livers with aberrant left hepatic artery. All of them have been reconstructed on bench table. There was no incidence of HAT in the entire cohort with ALHA. The one-year graft survival for this patient cohort was $93.1 \%$. Our surgical approach resulted in a low incidence of HAT of $1 \%$ with excellent graft survival.
\end{abstract}

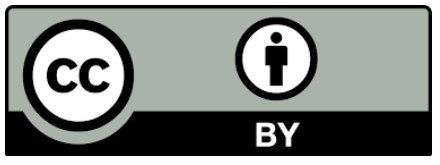

(C) 2021 by the author. This is an open access article distributed under the conditions of the Creative Commons by Attribution License, which permits unrestricted use, distribution, and reproduction in any medium or format, provided the original work is correctly cited. 


\section{Key words}

Liver transplantation; arterial hepatic reconstruction; hepatic arterial thrombosis

\section{Introduction}

One of the most feared complications in liver transplantation is hepatic arterial thrombosis (HAT). The incidence of HAT in liver transplantation varies from $1.2 \%$ and $8 \%$. One of the risk factors for this complication is anatomical complexity of hepatic arterial system. Anomalous arterial anatomy exists in 20 to $40 \%$. The aberrant left hepatic artery (ALHA) arises from the left gastric artery, with a prevalence of $13 \%$. There is also geographic variation in the prevalence of this anomaly (14\% in the Europeans, $6 \%$ in the Asians, and 4\% in South Americans). As per Michel's classification [1], the ALHA can be a) the only variation (Type II) (3.7\%) or b) in association with an aberrant right hepatic artery (Type IV, 3.2\%).

The focus of this letter is on the surgical reconstruction of aberrant left hepatic artery (Type II \& IV of Michel's classification). While there is a consensus on reconstruction of an aberrant right replace artery (Type III), there is still a debate when it comes to ALHA. The ALHA can be of two types - replaced artery (supplying all of the left lobe and accessory artery (supplying to a part of the left lobe). Unfortunately, during liver procurement it is difficult to know which of these 2 different categories the ALHA is since the intrahepatic branches cannot be explored. This is even more difficult during donation after cardiac death procurements. Procurement injuries to the aberrant vessels are unfortunately common and can lead to graft loss [2]. At times, injury done during the organ procurement to ALHA is the sole reason for liver discards. Different centers have described surgical approaches to an ALHA. Montalti et al [3] describe a way to decide if ALHA vessel is replaced (needs to be preserved) or an accessory (can be ligated). Their approach is slightly complicated and involves both phases of liver transplant - procurement and liver implantation. When there is no injury to the ALHA, it can be incorporated in the arterial anastomosis by using donor celiac artery for the arterial anastomosis. However, this can bring redundancy to the hepatic artery which can lead to kinking. When there is injury to the ALHA, some type of reconstruction is required, often to the gastroduodenal artery (GDA).

In this letter, we describe our approach to an ALHA. At our center, it has been our routine practice is to keep the arterial anastomosis straight and short to avoid kinking and thereby HAT. With the standard hepatic arterial anatomy, our typical anastomosis is between the donor hepatic artery just proximal to the GDA to the recipient hepatic artery distal to the GDA. We believe this technique lowers the incidence of HAT [4]. To achieve this "short and straight" arterial reconstruction, we prefer to reconstruct the ALHA to the GDA routinely, unless it is very small (less than $3 \mathrm{~mm}$ ). Very small ALHA is usually left intact, and in this case the main hepatic artery is shortened between GDA and left gastric artery to avoid redundancy. All arterial reconstruction has been done end to end avoiding abrupt vessel angulation. All ALHA bigger than $1 \mathrm{~mm}$ has been reconstructed. 


\section{Surgical Technique}

\subsection{Type I Reconstruction: ALHA to GDA Reconstruction}

In most cases the GDA was used to reconstruct AHLA. The anastomoses was performed between the stump of the GDA to either the ALHA or its cuff off of the left gastric artery in an end to end fashion. All anastomosis were performed using 8-0 or 7-0 Prolene suture using interrupted or running fashion depending on the caliber of the vessels. $5 \mathrm{Fr}$ pediatric feeding tube was used as an intravascular tutor.

\subsection{Type II Reconstruction: Shortening of the Main Hepatic Artery (MHA)}

Whenever the diameter of AHLA is less than $3 \mathrm{~mm}$ and safe reconstruction was difficult, the AHLA along with the left gastric artery (LGA) was kept intact. In these situations the MHA was shortened. In this type of reconstruction a segment of MHA was cut between the origins of the LGA and the GDA and an anastomosis the cut ends were simply anastomosed in an end to end fashion with 7-0 Prolene in running fashion. This reconstruction could be performed after implantation of the liver and if indeed there is kinking of the MHA (as described by Montalti). However, when MHA is clearly too long, it is advantageous to perform this anastomosis on the back table as it becomes easier and avoids arterial re-clamping.

\subsection{Type III Reconstruction: ALHA to GDA + Reorientation of the Proper Hepatic Artery}

In cases where the GDA is close to the bifurcation of proper hepatic artery into the left and right hepatic arteries, the ALHA to GDA reconstruction can cause a 90-180 degrees twist and pose a risk of narrowing and HAT. To avoid this, after the ALHA-GDA anastomosis the proper hepatic artery is divided distal to the GDA and re-anastomosed with a 90 - 180 turn of the MHA to the right. This type of reconstruction is performed only when the size of proper hepatic artery is not too small.

\section{Results}

Between January 2016 and June 2019, we procured and transplanted 357 adult liver allografts. Of these, there were 34 (9.5\%) livers with AHLA. Of those 34 livers, back table reconstruction to the GDA was performed in 25 (70\%) [Type I reconstruction], hepatic artery was shortened in 5 (20\%) [Type II reconstruction], and 2 (5\%) were left as is. In 2 (5\%) in presence of an injury to the LGA, ALHA to GDA reconstruction was performed, however this was associated with a significant twist at the level of proper hepatic artery distal to the GDA. To negate this twist and the risk of HAT, we performed an additional anastomosis at proper hepatic artery [ Type III reconstruction] after undoing the twist. Retrospectively the incidence of HAT and liver graft survival were assessed. Routine doppler ultrasound exams were performed on post-transplant day 1 in all cases. HAT was identified by a doppler ultrasound or a contrast CT scan. There was no incidence of HAT in the entire cohort with ALHA. The one year graft survival for this patient cohort was 93.1\%. At our center, the incidence of HAT in last 1145 adult liver transplants is $1 \%$ [3]. 


\section{Discussion}

This manuscript describes a surgical technique that was routinely employed to allow short length of hepatic artery during liver transplantation. This approach resulted in a low incidence of HAT of $1 \%$ with excellent graft survival. Keeping the donor MHA short has worked well for us and has provided excellent outcomes [4]. For this reason, it is our preference to reconstruct ALHA to GDA. However, many centers routinely perform arterial anastomosis at the level of donor celiac artery, in which case this type of reconstruction is unnecessary. However, we know from experience that there are situations where arterial anastomosis at the level of celiac artery is not always possible. For instance, in donors with atherosclerosis, it is not uncommon to find arterial plaque at the levels of celiac artery, origin of LGA and splenic artery. Additionally, procurement injuries to ALHA are not uncommon, traction injury being the common mode of injury to ALHA. During DCD procurements, ALHA may not be identified with impunity and thereby divided inadvertently. In order to make such liver graft implantable in abovementioned circumstances, it becomes necessary to sacrifice the celiac artery and anastomosis at the level of MHA is the only option. The reconstruction techniques described here can be utilized in such situations. This reconstruction can also be useful when simultaneous procurement of a modified multivisceral graft is being pursued.

The limitation of this study is that is a retrospective. It is also showing our approach to preserve possibly all ALHA without trying to individuate if it is replaced or accessory.

In conclusion, livers with ALHA can be successfully transplanted with excellent outcomes. This study presents a method of ALHA reconstruction that allows short and straight arterial implantation, which in our experience is associated with a low incidence of HAT. Proficiency in this reconstruction may allow surgeons to deal with procurement injuries to ALHA and prevent organ discards.

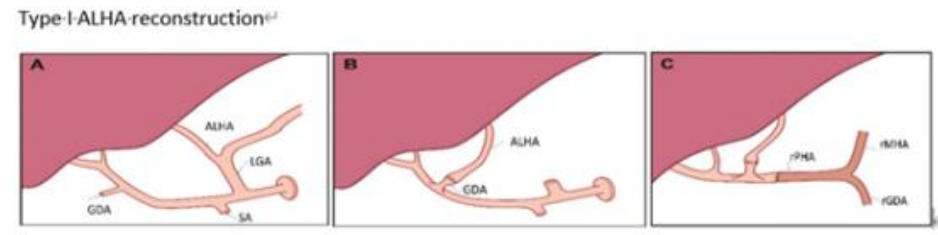

Type-II-ALHA-reconstruction

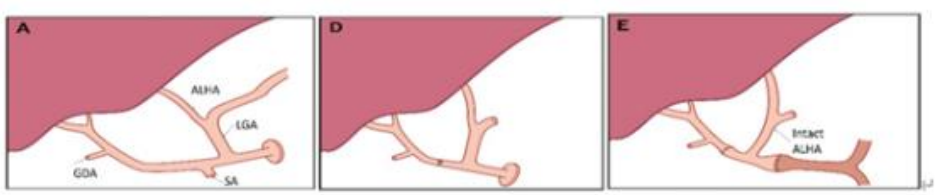

Type-III-ALHA-reconstruction

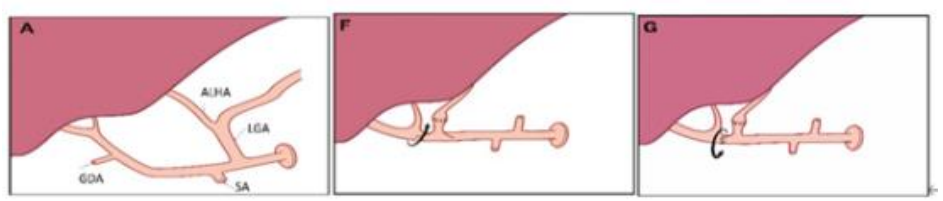

Figure 1 Top panel represent Type I reconstruction (ALHA to GDA). Middle panel represents type II reconstruction (shortening of main hepatic artery) and lower panel represents type III reconstruction (undone the twist created after Type I reconstruction). 


\section{Abbreviations}

HAT - hepatic arterial thrombosis; ALHA - Aberrant left hepatic artery; GDA - gastro-duodenal artery; MHA - main hepatic artery; LGA - left gastric artery; SA - splenic artery.

\section{Author Contributions}

Plamen Mihaylov, Burcin Ekser, Daiki Soma - writing, data collection and final approval; Andrew Lutz, Jonathan Fridell - analysis and interpretation of data for the work; Shekhar Kubal - revising manuscript.

\section{Funding}

The authors declare no funding was received for this study.

\section{Competing Interests}

The authors declare no conflicts of interest.

\section{References}

1. Michels NA. Blood supply and anatomy of the upper abdominal organs with a descriptive atlas. Philadelphia: J. B. Lippincott Company; 1995.

2. Yu YD, Kim DS, Byun GY, Suh SO. Liver abscess developed after cadaveric liver transplantation due to ligation of an accessory right hepatic artery of the donor graft. J Korean Surg Soc. 2012; 83: 246249.

3. Montalti R, Benedetti Cacciaguerra A, Nicolini D, Ahmed EA, Coletta M, De Pietri L, et al. Impact of aberrant left hepatic artery ligation on the outcome of liver transplantation. Liver Transplant. 2018; 24: 204-213.

4. Schroering JR, Kubal CA, Hathaway TJ, Robinson RC, Mangus RS. Impact of variant donor hepatic arterial anatomy on clinical graft outcomes in liver transplantation. Liver Transplant. 2018; 24 : 1481-1484. 
OBM Transplantation 2021; 5(1), doi:10.21926/obm.transplant.2101138

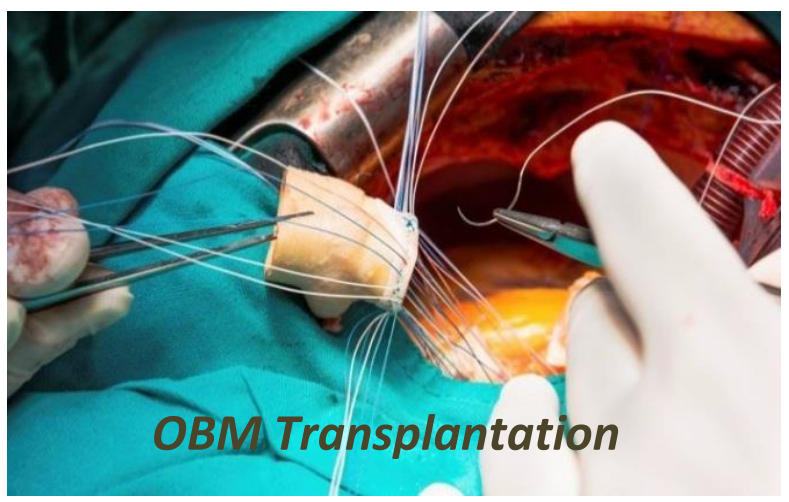

Enjoy OBM Transplantation by:

1. Submitting a manuscript

2. Joining in volunteer reviewer bank

3. Joining Editorial Board

4. Guest editing a special issue

For more details, please visit:

http://www.lidsen.com/journals/transplantation 